\title{
Realization of CyberRail Partial Functions in Actual Environment
}

\author{
Takahiko OGINO \\ Supervisor Researcher, Transport Information Technology Division
}

Ryuji TSUCHIYA

Laboratory Head, Passenger Information Systems Group, Transport Information Technology Division

\begin{abstract}
CyberRail is an information technology (IT)-based infrastructure or system concept on which a new generation of railway services is founded. In other words, each railway operator will be able to develop its own new business model based on it to improve the quality of railway transport, which will let railway business regain a pivotal position in world mobility. To realize this objective, there are two basic factors: travel demand and the more difficult pinpointing of passenger locations at any given time. This paper explains a trial approach to realize a partial realization of CyberRail functions, particularly from the viewpoint of how to track passenger locations.
\end{abstract}

Keywords: CyberRail, location, travel demand, ubiquitous, AFC gate, goopas

\section{Introduction}

People make use of transport services. Although this is a very apparent fact, researchers and planners for transport services in roads and railways tend to start from the car or train's perspective instead of from that of the people: the former are just means of transport, the latter are end users to be transported. How to provide people with appropriate information is becoming more important, in order to guide, protect, and make people as comfortable and relaxed as possible when they use transport facilities, to which the lowest priority is unfortunately given. When we reconstruct transport systems, we have to start from the passenger's viewpoint.

In different regions, operators and manufacturers construct their own unique systems in accordance with their own needs, business activities, profit levels, etc. This may create a situation where a journey through these transport services is not so convenient as compared with each individual ride. One solution to cope with this situation and unite these systems is to introduce intermodal transport, the aim of which is to facilitate efficient and convenient use of compatible transport modes. The CyberRail research project is a softwarebased intermodal concept that aims to improve transport efficiency and convenience. Software offers a variety of options for supporting intermodal transport.

When various independently developed systems, such as automobiles, railways, and airplanes, are reviewed from the viewpoint of user interoperability (meaning how easily passengers can travel through all transport modes), barriers have arisen that make it inconvenient for passengers, that is, that force them to change from one transport mode to another, until a globally unified hardware system can be offered, which would be expensive, inefficient or difficult to realize. Under these circumstances, it is most important to establish a general system or a mechanism with sufficient flexibility and openness for the various anticipated systems to assure user interoperability, while meeting the individual re- quirements of operators located at various places and without undermining the principles of competition. In this context, it is becoming more and more important to develop software-based interoperability technologies that can meet new social needs by fusing various hardware solutions into one comprehensive and effective whole.

\subsection{Differences from a passenger guidance system}

Conventional rail transport consists of the following actors:

- Passengers taking trains which operate and roughly meet their potential travel demands

- Railway operators running trains to a published schedule and maintaining railway-related equipment and facilities, provided that the demands of passengers are the same as the planned schedule

One of the reasons why CyberRail is so different from an information provision and guidance system is that it aims to create a new transport capacity such as running extra trains or increasing the number of coaches, depending upon the current demands from passengers, which are taken into the system through interfaces with information guidance. CyberRail also aims at giving passengers information in order to optimize passenger flows.

Under this new method of control, there are no distinctions between normal and abnormal train control methods, although there are normal and abnormal passenger flows. The new method optimizes train movements in order to adjust passengers' demands in any situation. This function is not only convenient for passengers, but also profitable for railway operators, because they could provide efficient transport capacity.

Communication between passengers and railway operators is an essential part of CyberRail. If information about what is going on in the real transport system were to be made available in cyberspace, we could introduce various types of planning methodology to solve ineffectiveness in transport operation control problems. This is why information processing is useful for creat- 


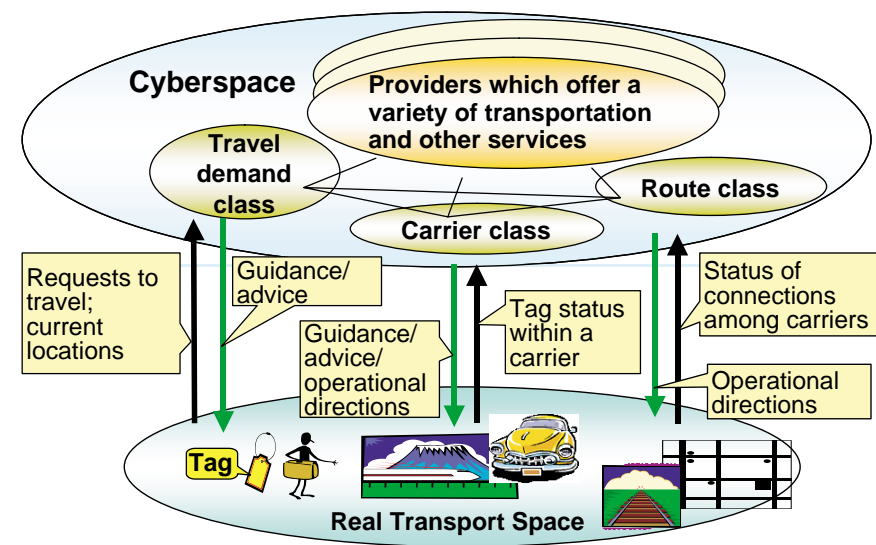

Fig. 1 Conceptual model of CyberRail

ing effective transport plan. This is explained in Fig. 1.

\subsection{Why CyberRail has been studied}

The study of CyberRail arose from various needs. It is expected that IT, the central technologies of which are ubiquitous and mobile communication, will reform the railway business model, which has remained essentially unchanged for 100 years, in terms of railway operators' management policy. From this, it is necessary to demonstrate a scenario in a new railway business model, or to make a fundamental concept model showing the information infrastructure on which Japanese and world transport systems can be built.

In other words, CyberRail is a study of the foundation, from the information science viewpoint, on which each railway operator can develop its own new business model to improve the quality of railway transport, which will give again the railway business a pivotal position in world mobility.

\section{Realization of Partial Functions}

\subsection{Goopas}

We have developed an experimental system that implements part of CyberRail's fundamental functions. To bring CyberRail services to fruition, it is very important to know the current location of each passenger during his/her journey. In the experimental system, we utilize the data from automatic fare collection (AFC) gates as the source of passenger location and timing information, entering or exiting a station. Other locations but data from AFC are estimated according to the train timetable.

Most of the railway stations located in urban areas in Japan are equipped with AFC gates, which are (or could be) connected to central computers. A private railway company in the Tokyo Metropolitan Area has started a new passenger service called "goopas" (Good Passport/ Good Passgate) that aims at promoting railway utilization. The outline of the service is as follows:

Passengers who want to use the new service must have a cellular phone that can receive electronic mails.
The identification numbers of passenger season tickets are registered in the host system of the service. Every time a registered passenger goes through an AFC gate, the ID number recorded in his/her ticket is sent to the host system, which can identify the current location and direction in which the passenger is traveling (entering or exiting a station). The system sends an electronic mail to the cellular phone of the passenger, which contains location-sensitive information tailored to fit the interests of the passenger. This includes information on nearby shops, restaurants, some town events, daily news, or other short stories of the day. Passengers can enjoy these location-sensitive advertisements delivered by the email from goopas free of charge since all advertisements are sponsored (See Fig. 2).

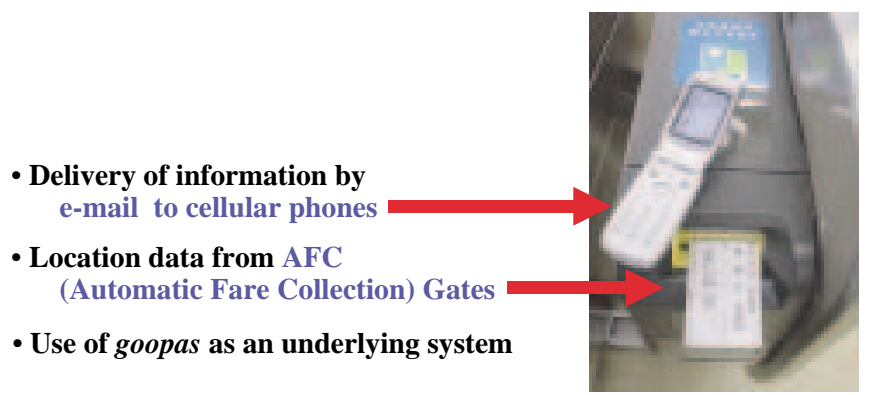

Fig. 2 AFC gate, cellular phone and season ticket

\subsection{Experiment Details}

The realized partial functions of CyberRail in the experiment are as follows:

(1) This service starts with consultation/guidance concerning a customer's trip, which is a very unique aspect of the CyberRail user interface. The system does not require users to input data, instead the system acts as a consultant giving users appropriate travel information. To receive appropriate information about his/her travel, data concerning origin/destination and other details are necessary and will be input unurged by the user through an Internet terminal or mobile phone.

(2) The system compiles alternatives for the passenger using a timetable inquiry system already on the Internet. CyberRail does not recommend that each rail company make all the subsystems themselves.

(3) The system sends back the alternatives to the passenger, one of which one can be selected. At this moment, the user interface is not sufficiently userfriendly. However, users can use the consultation mode as much as they want.

(4) Once the user decides on a plan, the system registers the selected itinerary data.

(5) The system sends back the itinerary and relevant information such as the maps of the departure station to the passenger as registration confirmation. All the functions explained up to this time can be used by passengers at anytime before the departure, a day before the departure, one month before, etc.

(6) On the day of departure, the system reminds the passenger to leave home or his/her office to catch the 
train in the itinerary $\alpha$ minutes before the train is due to leave.

(7) If the passenger fails to pass through the ticket gate $\beta$ minutes prior to the departure time, the system sends a warning message together with the next possible itinerary.

(8) There are two scenarios. One is that the passenger takes the original train, the other is that the passenger fails to catch it.

(9) The system can decide which scenario happens according to the time the passenger passes through the gate. That is, if this is less than $\delta$ minutes prior to departure, the system decides that the passenger is late. As this is rather indirect, the system could be improved if the position of the passenger were to be traceable at any time.

(10) If the passenger fails to catch the first train and catches the second one, the system guides the passengers based on the second itinerary.

(11) In this experiment, if the passenger fails to catch both of these trains, the system decides that the passenger has abandoned the journey.

(12) The system informs the passenger of which itinerary is to be taken, when he/she passes the gate at the departure station.

(13) Just $\gamma$ minutes before the arrival at the transfer station, a reminder mail arrives.

(14) Just $\gamma$ minutes before the arrival at the destination station, an arrival notice will also be received.

(15) When the passenger passes through a ticket gate at the destination station, information about the final destination may be sent to the passenger and the session is closed.

We utilise goopas as the underlying system of the experimental CyberRail sub functions. Although the passengers' location data are available only when passengers go through AFC gates (in or out), it is better than nothing. In addition, the system predicts the location of a passenger based on timetable information (i.e. train schedules) and the passenger's itinerary so that we can plot when the passenger is approaching a transfer station or the final destination. Fig. 3 shows the timing chart and Fig. 4 also explains the structure of the experimental system. Of course it is desirable to utilize

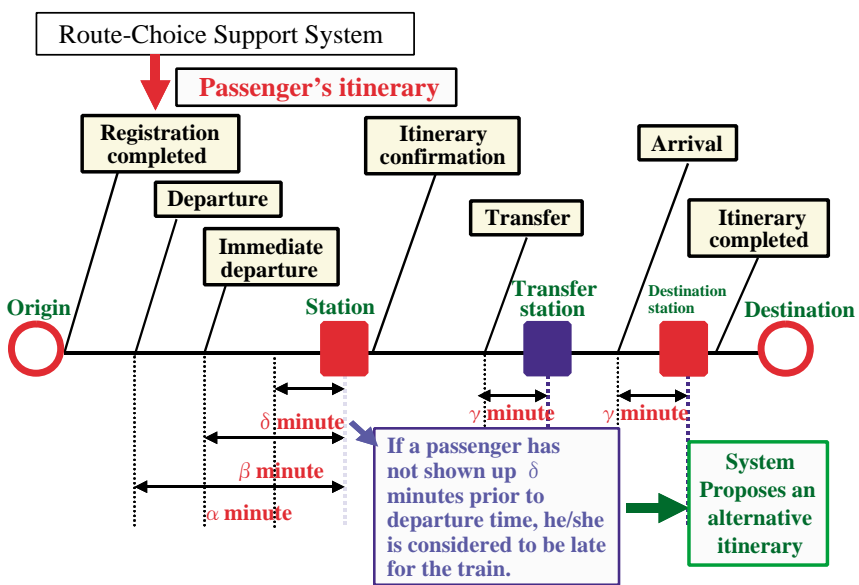

Fig. 3 Message timing chart

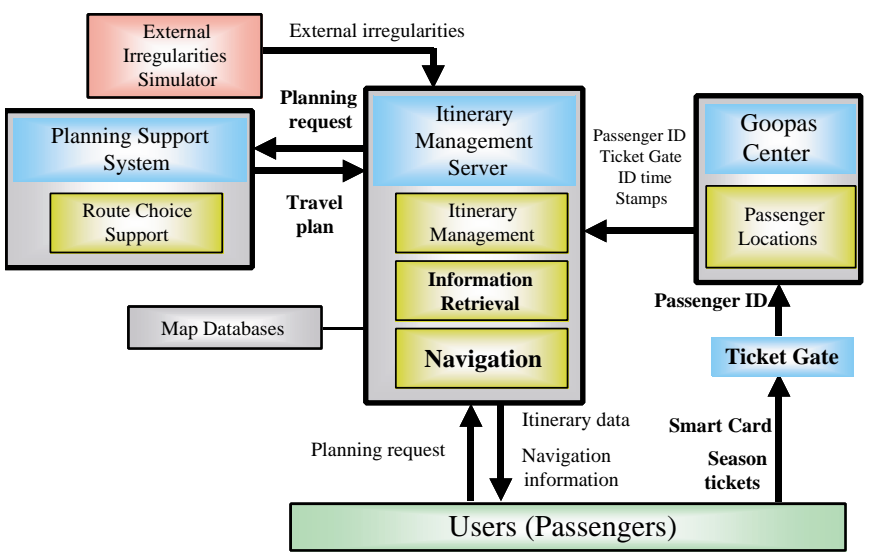

Fig. 4 Structure of experimental system

other mechanisms to obtain more precise and detailed passenger location data (GPS, etc.) for the real CyberRail services.

\subsection{Experiment Evaluation}

Experimental operation of the system has been carried out by the Railway Technical Research Institute, OMRON Corporation, the goopas system manufacturer, and Odakyu Electric Railway Co. Ltd., one of the major private rail companies in the Tokyo urban area. From August 2003 through March 2004, 103 users used this system and 67 of them have responded to the questionnaire which asked if the system is necessary, its effectiveness, suitability of the contents and the distributed timings, etc.

The questionnaire survey showed that many users highly rated the service provided by the system and that most of them were satisfied with the contents of distributed messages (Figs. 5 and 6).

Some people commented that the system is effective enough to be able to support novice passengers who do not use railways frequently. One of the features which was very much appreciated by the users was the system's ability to flexibly adjust passengers' itineraries. The survey also revealed that they have strong needs for large-scale information and guidance at complicated stations where they often find difficulties in getting to the right platform. It was also pointed out by a number of users that information delivery by e-mail to cellular phones is not dependable because there might be some delivery delays due to communication network conges-

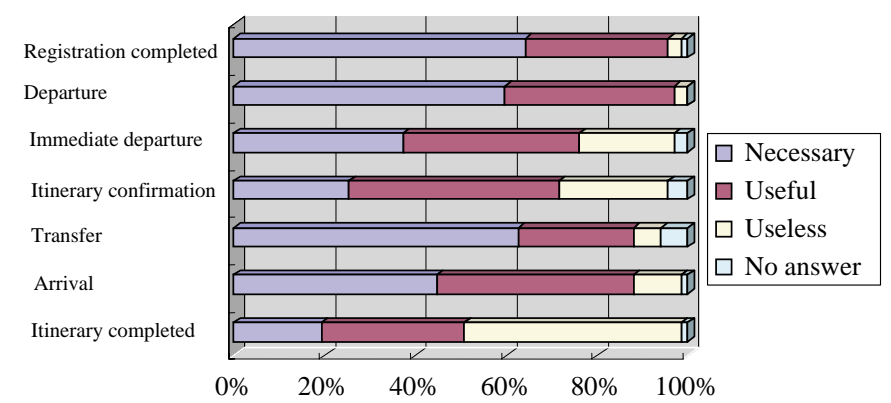

Fig. 5 Necessity of delivered messages 


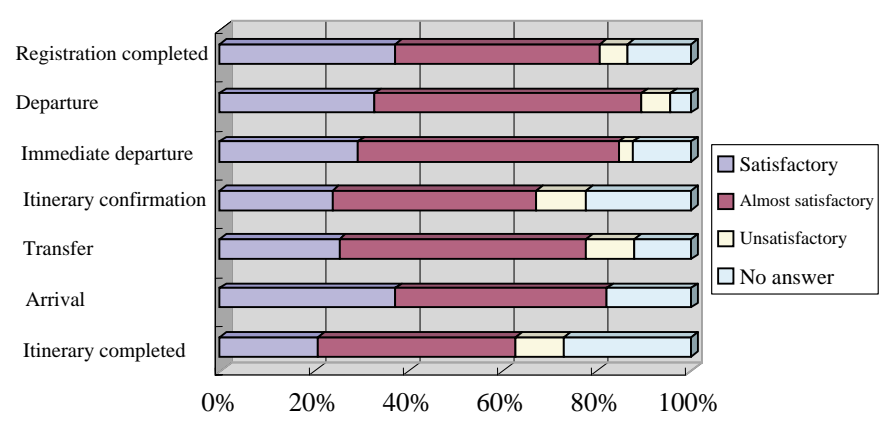

Fig. 6 User satisfaction with delivered messages

tion or mail server overload.

\section{Future of CyberRail Research}

From this experiment, we had confidence that the system would be welcomed in the near future. However, from the engineering viewpoint, this is only the starting point of CyberRail research. We still have the following research themes to pursue:

\subsection{Railway business related}

\section{(1) Modes Transfer Guidance System}

As mentioned above, journey guidance or consultation is one of the core functions of CyberRail. Although a number of train transfer guidance systems are proposed, little progress has been made with such facilities that take into account personal preference and requirements. Examples of specific requirements are:

- Transfer guidance using only stations equipped with escalators/elevators

- Adjustable transfer time from one train to another, according to individual walking abilities and station congestion

- Platform number guidance, etc.

These requirements can be met not only by improving software engines, but also by providing appropriate data. This leads to the next study theme.

\section{(2) Standardization of timetable and guidance infor- mation}

One of the difficulties in standardizing timetable and guidance data is the differences in their forms among various railway operators and transport companies. This is because of the traditions and technical developments in each organization, due to the differing passenger flows in areas, and different operating environments.

On the other hand, the data describing the current traffic situation is very important, particularly from the viewpoint of passenger guidance. A railway company needs other companies' data, because passengers might reach their final destination using other companies' services. A standardized representation of this data, different from their current form and that uses the least conversion processing, is needed.

It is thought that Web service technology could be appropriate for this purpose. Although format standardization of the original data that is actually used by each company would be difficult, if they have a Web Services Description Language (WSDL) to describe the interface for data access, they could share the information with each other. Moreover, it would be easier to have a standard WSDL for data access than to standardize the raw data format.

\section{(3) International Standardization of Tickets and their Settlement}

CyberRail does not introduce a ticket concept, because the tariff can be calculated from the trace data of a passenger. In reality, however, we have to introduce tickets, though they may be in the form of IC or in the Internet. Handling tickets, particularly in foreign countries, is troublesome. We have to solve this problem from an international viewpoint.

One comment, if we could mention it, is the differing situation in Asian countries, where, unlike Europe and USA, many people use trains, fare systems are complex, ticket gates are usual, and heavy penalties for traveling without a ticket are unpopular. Therefore, we have to consider these differences when a standard for handling tickets or fares is introduced.

\subsection{Railway management}

\section{(1) Timetable planning, operation and rescheduling} based on passenger demand

This is a very fundamental problem, which prompted the initial discussion of CyberRail several years ago. The basic idea is as follows. If railway operators had bidirectional communication links to passengers, old style reservations would not be necessary (see Fig. 7).

Travel planning is like a golf game. You do not have to make a precise shot at the start. Instead, an almost precise and long carry is needed. In the case of travel planning, the booking of the date/time-interval of departure/arrival, departure and arrival stations would be necessary when you decide on a travel plan, say one month in advance.

In other words, a traveler gets a guaranteed seat to carry him/her from the departure station to the destination around the time specified. Fundamentally, this is a sufficient requirement from the passenger viewpoint, and a very convenient specification for railway operators, because, by means of this system, operators can interpret the booking of a seat as placing a space request on a travel demand queue. Operators have the freedom to set up a train maximizing passenger satisfaction requests, that is, the train might not be exactly what one specific person wants, but many passengers will be satisfied with it.

One of the reasons why we could not introduce this system before is that we did not have a cheap and easy two-way communication tool. Now we have cellular phones or easy access to the Internet, by which railway operators provide passengers with detailed information at a later stage, or even negotiate passengers requests.

Once a train schedule and other conditions have been fixed, by using the abovementioned two-way communication channel, precise information on seating would be provided. 


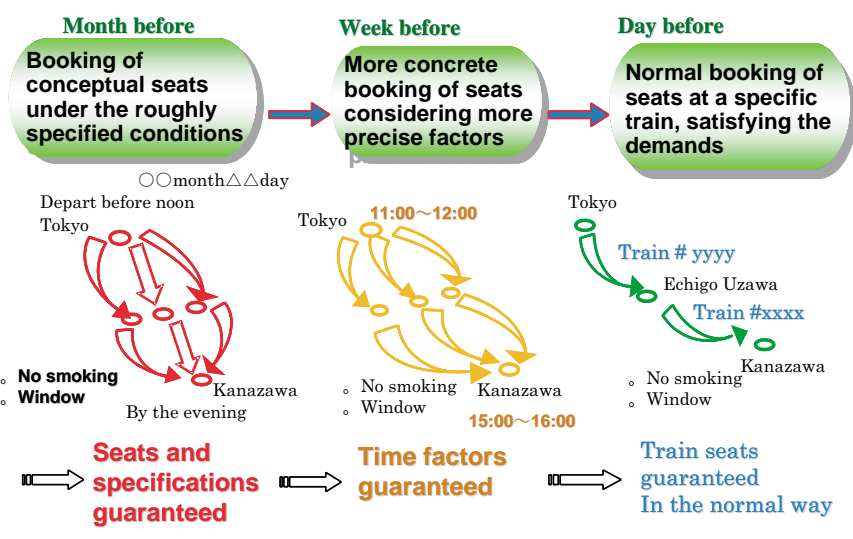

Fig. 7 Booking by bi-directional communication

In Fig. 7, a passenger books a virtual train on the routes shown. A week before the departure, a more concrete form of train information will be sent. Then finally, just before the departure, the passenger will get the detailed information, possibly with additional information on how to reach the departure station, etc.

\subsection{Formal Methods and Railway Domain Model}

RTRI is promoting research into formal methods to improve the reliability of software for safety critical systems. A reliability, availability, maintainability and safety (RAMS)-related norm (IEC62279) recommends the use of formal methods to guarantee the safety of software whose safety integrity level (SIL) is four. Causes of failure in software are called systematic failures, which are different from random failures, because they will always occur under certain conditions. In addition, it is very difficult to find these conditions.

Generally, so-called debugging is a way to minimize potential failures, but we cannot get a guarantee that there will be no more failures in the software in this way. One small failure, affecting a very small proportion of software, will cause a catastrophe if the situation persists. This is totally different from random failures.

One of the applications for formal methods is a domain model approach. A domain model can be expressed as a compressed form of knowledge and know-how in the technology domain. It can be used as a reference model for newly building a system and used an encyclopedia of the past knowledge and know-how. Ohm's law and Maxwell's equations in electric and magnetic engineering are considered as examples of domain models. In the case of software, domain models would not be in the concise form like these.

From the model-based approach, the domain model in software technology can be expressed as a data structure, data-related invariants or axioms, and abstract procedures written in a formal language. Although it is currently at a research level centering on universities, the standardization of software may be realized in this way. For details, please refer to http://www. railwaydomain.org/.

\section{Conclusion}

The study of CyberRail started in 1999 from a proposal for a passenger guidance system, in the search for the intermodal transport system of the future. Then the idea of creating new traffic capacity according to passenger demand followed. To solve problems about restrictions in traffic or train planning diagrams, consideration was given to both carrier and route classes. Finally, the idea of controlling disruptive factors became one of the objectives of the CyberRail study.

After the completion of CyberRail, the disruptive factors can be cooperated on or mitigated into railway operators management cycles. We believe that railway transport quality, safety in particular, can be achieved only by a total approach consisting of research, business management, information sharing, etc., based upon an appropriate model or CyberRail architecture.

\section{References}

1) http://cyberrail.rtri.or.jp/english/

2) Takahiko OGINO, "When Train Stations become CyberRail Stations," Japanese Railway Technology Today, 209-219, December 2001, ISBN4-330-672014, Transportation News Co., Ltd., Japan.

3) Takahiko OGINO, Yasuo SATO, Kiyotaka SEKI, Ryuji TSUCHIYA, "CyberRail - the fabric of ITS enhanced by railway transport-," Proc. of the World Congress on Railway Research 2001 (WCRR2001).

4) Kiyotaka SEKI, Ryuji TSUCHIYA, Takahiko OGINO, Yasuo SATO, "Construction of railway system utilizing information and communication Technologies," Proc. of the World Congress on Railway Research 2001 (WCRR2001).

5) Ryuji TSUCHIYA, Kiyotaka SEKI, Takahiko OGINO, Yasuo SATO, "CyberRail: An Enhanced Railway System for Intermodal Transportation," Proc. 8th World Congress on Intelligent Transportation Systems.

6) Ryuji TSUCHIYA, Kiyotaka SEKI, Takahiko OGINO, Akihiko MATSUOKA, "Deriving interoperable traveler support system specifications through requirements engineering process," Proc. of the 7th World Multiconference on Systemics, Cybernetics and Informatics (July 2003).

7) Takahiko OGINO, Koichi GOTO, Ryuji TSUCHIYA, Kiyotaka SEKI, Akihiko MATSUOKA, "CyberRail and its significance in the coming ubiquitous society," Proc. of the World Congress on Railway Research 2003 (WCRR2003).

8) Ryuji TSUCHIYA, Akihiko MATSUOKA, Koichi GOTO, Kiyotaka SEKI, Takahiko OGINO, "Requirement-driven Approach to Interoperable Traveler Support System Specification," IEEE 12th International Conference on Requirements Engineering, 2004.

9) Ryuji Tsuchiya, Akihiko Matsuoka, Koichi Goto, Takahiko Ogino, "Design and Implementation of Context-Aware Passenger Support System," 11th World Congress on ITS, 2004. 